\title{
A SIMPLIFIED METHOD FOR UPSCALING COMPOSITE MATERIALS WITH HIGH CONTRAST OF THE CONDUCTIVITY
}

\author{
R. EWING*, O. ILIEV†, R. LAZAROV ${ }^{\ddagger}$, I. RYBAK ${ }^{\S}$, AND J. WILLEMS $₫$
}

\begin{abstract}
An efficient approach for calculating the effective heat conductivity for a class of industrial composite materials, such as metal foams, fibrous glass materials, and the like, is discussed. These materials, used in insulation or in advanced heat exchangers, are characterized by a low volume fraction of the highly conductive material (glass or metal) having a complex, network-like structure and by a large volume fraction of the insulator (air). We assume that the composite materials have constant macroscopic thermal conductivity tensors, which in principle can be obtained by standard up-scaling techniques, that use the concept of representative elementary volumes (REV), i.e. the effective heat conductivities of composite media can be computed by post-processing the solutions of some special cell problems for REVs. We propose, theoretically justify, and numerically study an efficient numerical method for computing the effective conductivity for media for which the ratio $\delta$ of low and high conductivities satisfies $\delta \ll 1$. In this case one essentially only needs to solve the heat equation in the region occupied by the highly conductive media. For a class of problems we show, that under certain conditions on the microscale geometry, the proposed method produces an upscaled conductivity that is $\mathcal{O}(\delta)$ close to the exact upscaled permeability. A number of numerical experiments are presented in order to illustrate the accuracy and the limitations of the proposed method. The applicability of the presented approach to upscaling other similar problems, e.g. flow in fractured porous media, is also discussed.
\end{abstract}

Key words. effective heat conductivity, numerical upscaling, fibrous insulation materials, metal foams, permeability of fractured porous media.

AMS subject classifications. 80M40, 80M35, 35J25, 35R05, 76M50

1. Introduction. The upscaled properties of composite materials/media, such as effective heat or electrical conductivities of compound materials, the effective permeabilities of porous media, etc. are in strong demand in engineering, geoscience, and environmental studies to name just a few. Below, we will mainly discuss calculating the effective heat conductivity. Note that electric conductivity, and meso- to macro-scale upscaling in saturated porous media, lead to the same mathematical problem.

It is well known, that for heterogeneous media, for which the heterogeneity length-scale is small compared to some macroscopic length-scale, it is often possible to extract some effective property describing the media on the macroscopic length-scale. The mathematical framework in which this determination of effective properties is carried out is the theory of homogenization. The cases when the small scale heterogeneities are either periodic or statistically homogeneous have been studied in detail in homogenization theory, see, e.g. $[15,5,16,20]$ and the references therein. In both cases the effective properties can be deduced by solving suitable sets of "cell" problems on representative elementary volumes (REV). For periodic and statistically homogeneous structures, the periodicity cell and a sufficiently large (compared with the length-scale of the heterogeneity) sample, constitute an REV, respectively. For a discussion of the definition of an REV and for derivation and justification of the homogenization procedure, we refer the reader to $[15,7,13]$, and to the references therein.

In this paper we consider the following mathematical problem: In a bounded domain $\Omega \subset \mathbb{R}^{n}, n=2,3$, the function $u$ satisfies the boundary value problem

$$
\left\{\begin{aligned}
\nabla \cdot(K(\boldsymbol{x}) \nabla u(\boldsymbol{x})) & =0, & & \boldsymbol{x} \in \Omega \\
u(\boldsymbol{x}) & =g(\boldsymbol{x}), & & \boldsymbol{x} \in \partial \Omega,
\end{aligned}\right.
$$

where the boundary data $g$ and the coefficient matrix $K(\boldsymbol{x})$ are given.

We assume that this boundary value problem is a simplified model of the following engineering problem: The domain $\Omega$ is occupied by two material constituents $\Omega_{M}$ and $\Omega_{A}$, with substantially different

\footnotetext{
*Institute for Scientific Computation, Texas A\&M University, College Station, TX, 77843, USA

${ }^{\dagger}$ Fraunhofer Institut für Techno- und Wirtschaftsmathematik, Fraunhofer-Platz 1, 67663 Kaiserslautern, Germany (oleg.ilieveitwm.fraunhofer.de) and Institute of Mathematics, Bulg. Acad.Sci., Acad.G.Bonchev str., bl.8, 1113 Sofia, Bulgaria.

${ }^{\ddagger}$ Department of Mathematics, Texas A\&M University, College Station, TX, 77843, USA, (lazarov@math.tamu.edu) and Institute of Mathematics, Bulg. Acad.Sci., Acad.G.Bonchev str., b1.8, 1113 Sofia, Bulgaria.

$\S$ Universität Stuttgart, IANS, Pfaffenwaldring 57, 79569 Stuttgart, Germany (rybak@ians . uni-stuttgart. de).

IFraunhofer Institut für Techno- und Wirtschaftsmathematik, Fraunhofer-Platz 1, 67663 Kaiserslautern, Germany (joerg.willemseitwm. fraunhofer.de).
} 
thermal conductivities $K_{M}$ and $K_{A}$, respectively (the subscripts ${ }_{M}$ and ${ }_{A}$ refer to "metal" and "air", respectively). We consider the case

$$
K(\boldsymbol{x})= \begin{cases}K_{M}=1, & \boldsymbol{x} \in \Omega_{M} \\ K_{A}=\delta, & \boldsymbol{x} \in \Omega_{A},\end{cases}
$$

where $\delta \ll 1$. We refer to this large difference in the conductivities of the constituents as materials with high contrast of the conductivity. The analysis and the algorithm discussed in this paper could be easily extended to the case when $K_{M}=K_{M}(\boldsymbol{x})$ and $K_{M}(\boldsymbol{x})$ is a function bounded from above and below or a uniformly positive definite and bounded matrix in $\Omega$, independently of $\delta$. However, we prefer to illustrate the motivation and the performance of the proposed method on this simple model problem.

In the typical case that we are interested in, the domain $\Omega$ along with the coefficient $K$ represents a very complex structure of a heterogeneous medium, for which the length-scale of the heterogeneity is sufficiently small compared to the length-scale of $\Omega$, i.e. $\Omega$ is an REV. It is then practical to model this medium, which is heterogeneous at microscale, as homogeneous at macroscale with an effective thermal conductivity tensor $\tilde{K}$, that is constant over $\Omega$. For simplicity let us assume, that $\Omega$ is brick shaped, and that its faces are parallel to the coordinate planes.

It is well known that the effective thermal conductivity tensor $\tilde{K}$ of $\Omega$ can be obtained by the following procedure (see, e.g. $[15,20,16,13])$ : Find $n$ linearly independent solutions $u_{i}, i=1, \ldots, n$ of

$$
\left\{\begin{array}{rlll}
\nabla \cdot\left(K \nabla u_{i}\right) & =0, & & \text { in } \Omega \\
u_{i} & = & x_{i} & \text { on } \partial \Omega,
\end{array}\right.
$$

where $x_{i}$ is the $i$-th component of $\boldsymbol{x}=\left(x_{1}, \ldots, x_{n}\right)$ and then compute

$$
\tilde{K} \boldsymbol{e}_{i}=\left\langle K \nabla u_{i}\right\rangle_{\Omega}:=\frac{1}{|\Omega|} \int_{\Omega} K \nabla u_{i} d \boldsymbol{x},
$$

where $\boldsymbol{e}_{i}$ denotes the $i$-th unit vector and $|\Omega|=$ meas $(\Omega)$.

The Dirichlet boundary conditions in (1.3) are one of the several possible choices for such cell problems. For a discussion about the advantages and disadvantages of using various boundary conditions, e.g. periodic or combinations of Dirichlet and Neumann, we refer to [20,7].

The aim of this paper is to discuss, mathematically justify, and numerically test an efficient numerical method for calculating the effective thermal conductivities $\tilde{K}$ for a specific class of composite materials (these could be effective permeabilities of porous media as well). We assume that the composite materials/media are characterized by

- a high contrast of the conductivities of the constituents,

- a large (low) volume fraction of the poorly (highly) conductive constituent,

- the highly conductive constituent forming a network with complex (but known) internal structure.

Examples for such composite materials are some industrial metal and glass foams, fibrous metal and glass materials, mineral wool, and the like, which are widely used in insulation or in advanced heat exchangers (see, e.g. [9] and [17]). Another example are fractured porous media, where the connected fractures usually occupy only a small fraction of the domain (see, e.g. $[18,2,3,12]$ ). The detailed distribution of the highly conductive material in a given volume could be obtained using voxel representation of 3-D scans or by computer statistical generators. Examples of such materials are shown on Figures 1.1 and 1.2. The media shown in Figures 1.1(a) and 1.2(a) were generated by GeoDict2007 software ${ }^{1}$, while the geometries shown on Figures 1.1(b) and 1.2(b) were obtained using voxel representation of 3-D scans.

There is an extensive literature on analytical and numerical methods for calculating effective heat conductivities of composite materials by solving (1.3), e.g. [15, 5, 20, 16, 19]. A major issue in numerical computations is that high contrasts lead to ill-condioned matrices of the corresponding system arising in the discretization of the differential problem (1.3). In general, the condition number depends linearly on $1 / \delta$. Moreover, the complex topology of the highly conductive material makes a design of good preconditioners very difficult. Furthermore, for random high contrast media the sizes of the REV tend to be large, which leads to very large numbers of unknowns in the discrete system. For our particular class of high contrast

\footnotetext{
${ }^{1}$ For more information about this software we refer to the following webpage: www.geodict.com
} 


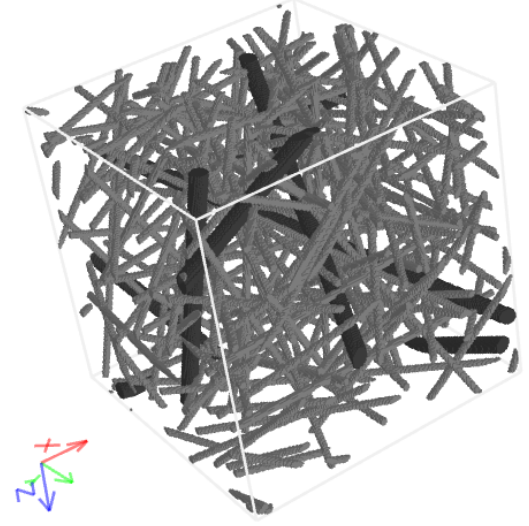

(a) Isotropic fiber structure.

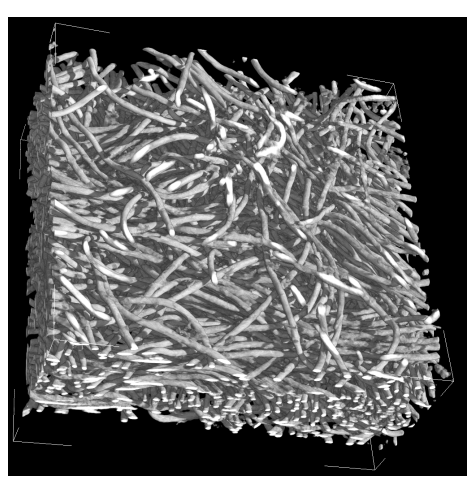

(b) Micro CT-scan of a fiber material.

FIG. 1.1. Examples of fibrous materials

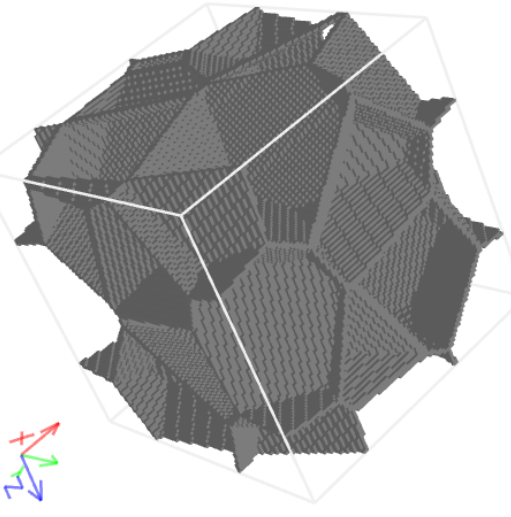

(a) Periodic foam structure.

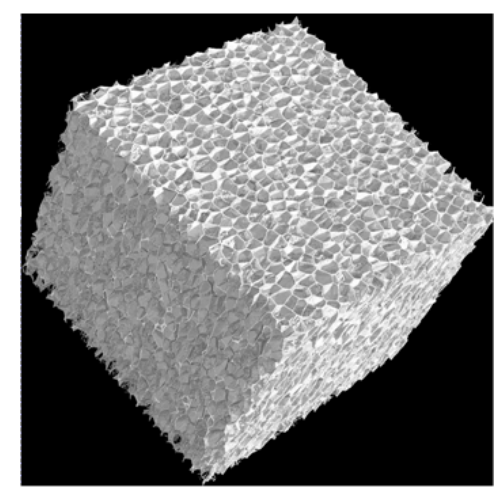

(b) Micro CT-scan of a foam.

FIG. 1.2. Examples of foam materials

materials we propose and justify a method that addresses both issues. The sizes of the algebraic systems are substantially reduced, namely to the fractions corresponding to the fiber contents, which for some industrial problems may lead to reductions to $1 \%$ of the initial unknowns, and the condition numbers of the corresponding systems are independent of $\delta$.

It should be noted that unlike in many other practical situations, we do not need the solution of (1.3), but we only need the functional defined by (1.4). Below we will show that in some cases $\tilde{K}$ can be approximated reasonably well by post-processing the solutions of constant coefficient problems, which are posed only on the part of the domain occupied by the highly conductive component.

The remainder of the paper is organized as follows: The motivation to replace the solution of (1.3) in the entire REV by solving a relevant problem in the subdomain occupied by the highly conductive material only is presented in Section 2. In Section 3 we give the theoretical justification of our approach. In particular we prove, that an $\mathcal{O}(\delta)$ approximation to $\tilde{K}$ can be computed in this way. The resulting algorithm, whose numerical complexity is independent of the contrast, is described in Section 4. The final Section 5 contains the results of selected numerical experiments confirming the theoretical derivations, as well as conclusions.

2. Notations and Motivation. We shall use the standard notations for Sobolev spaces of functions defined on $\Omega$ and its boundary $\partial \Omega$, respectively: $L^{2}(\Omega), H^{1}(\Omega), H_{0}^{1}(\Omega)$ etc. and the space $H^{\frac{1}{2}}(\partial \Omega)$ of traces of functions in $H^{1}(\Omega)$. Further, $\nabla v$ is the gradient of the scalar function $v, \nabla \cdot \mathbf{w}$ is the divergence of the vector function $\mathbf{w} \in \mathbb{R}^{n}$, and $\|\mathbf{w}\|$ will denote some vector norm of in $\mathbb{R}^{n}$.

By assumption the conductivity in $\Omega_{M}$ is much larger than the one in $\Omega_{A}$, i.e. $\delta \ll 1$. The proposed 
method relies on the following intuitive observations:

Firstly, the effective permeability $\tilde{K}$ is rewritten in the form

$$
\tilde{K} \boldsymbol{e}_{i}=\delta \frac{\left|\Omega_{A}\right|}{|\Omega|}\left\langle\nabla u_{i}\right\rangle_{\Omega_{A}}+\frac{\left|\Omega_{M}\right|}{|\Omega|}\left\langle\nabla u_{i}\right\rangle_{\Omega_{M}}
$$

so that in case $\nabla u_{i}$ on $\Omega_{A}$ is bounded independently of $\delta$, the first term is of order $\mathcal{O}(\delta)$ and can be neglected for high contrast materials.

Secondly, the normal temperature flux is continuous across the interface between $\Omega_{A}$ and $\Omega_{M}$ and therefore, intuitively, the normal component of the temperature gradient in $\Omega_{M}$ at the interface with $\Omega_{A}$ should tend to zero as $\delta \rightarrow 0$. Thus, it seems natural to approximate $\left.u_{i}\right|_{\Omega_{M}}$ by solving (1.3) in $\Omega_{M}$ by using homogeneous Neumann boundary conditions on the interface $\Sigma:=\partial \Omega_{M} \cap \partial \Omega_{A}$. Hence, we need to find $u_{i} \mid$ in a smaller domain $\Omega_{M}$. More important, $\left.u_{i}\right|_{\Omega_{M}}$ is the solution of an elliptic problem with constant coefficients which which leads to a much better conditioned discrete problem.

These two observations are the basis of our method. In the resulting algorithm described in Section 4, we also take into consideration some approximation of the contribution of the low conductive material on the overall effective thermal conductivity. This, however, is done cheaply in terms of numerical complexity. In particular, no additional discrete problem is solved in $\Omega_{A}$.

It should be noted that the idea of solving only in the highly conductive parts of the domain was proposed earlier in [4, pg. 105-106] in connection with models for flow in fractured porous media. In [4] there is, however, no rigorous justification, and the approximation suggested by [4, eq. III.90] for the permeability tensor still contains an undetermined geometrical factor. In [6] Bogdanov et al. also discuss the computation of effective permeability tensors for fractured porous media. For this the domain is decomposed into a highly permeable network (the fractures) and the low permeable surrounding media. Then the cell problems are separately solved in the network and in the background material. Thus, also the lowly permeable parts of the domain are accounted for. However, this happens at the expense of solving additional discrete problems. In section 4 we discuss how we account for the low conductive part of the domain without solving additional problems. Furthermore, one main objective of this paper is a rigorous mathematical justification of the approach of restricting to the highly conductive parts of the domain when calculating the effective conductivity tensor. In [6] such a rigorous discussion is not presented.

For the sake of completeness we would also like to mention that in [1] the idea of using zero Neumann boundary conditions on the interface between high and low conductive regions is used for the design of preconditioners for problems arising from discretizing equations like (1.3).

3. Analysis of the upscaling method for high contrast materials. This section contains the theoretical justification of the approach outlined above. First, Lemma 3.1 states an auxiliary result essentially saying, that the solution of (1.3) is bounded in $H^{1}$-semi-norm, independently of the contrast $\delta$. Next, Proposition 3.7 allows to neglect the highly conductive components which are not connected to the boundary of the domain, and Proposition 3.9 provides a way to approximate the average of the heat flux inside the remaining highly conductive components, i.e. those which are connected to the boundary.

Having proved these preliminaries, we are able to show our main result, i.e. Theorem 3.10, stating, that an $\mathcal{O}(\delta)$ approximation of $\tilde{K}$ can be obtained by post-processing the solutions of constant coefficient elliptic equations in the subdomain of the highly conductive material, subject to the linear drop Dirichlet boundary conditions on the outer boundary and zero Neumann boundary conditions on the interface between air and metal.

3.1. $H^{1}$-boundness of the solution independently of $\delta$. Now, we prove the following auxiliary lemma, that is a basis for the theoretical justification of our method:

LEMMA 3.1. Let $\Omega$ be a Lipschitz domain with $\Omega=\left(\bar{\Omega}_{M} \cup \bar{\Omega}_{A}\right) \backslash \partial \Omega$, where $\Omega_{M}$ and $\Omega_{A}$ are open sets with Lipschitz boundaries. Furthermore, let $u$ be the solution of (1.1), (1.2), where $g \in H^{\frac{1}{2}}(\partial \Omega)$. Then, for all $\delta$

$$
\|\nabla u\|_{L^{2}(\Omega)} \leq C,
$$

where $C$ is a constant which may depend on the domains $\bar{\Omega}_{M}$ and $\bar{\Omega}_{A}$, the properties of their boundaries, etc., but is independent of $\delta$. 
Proof. First, we find the functions $v_{M}$ and $v_{A}$ that solve the following problems, respectively:

$$
\left\{\begin{aligned}
\Delta v_{M}(\boldsymbol{x}) & =0, & & \boldsymbol{x} \in \Omega_{M} \\
v_{M}(\boldsymbol{x}) & =g(\boldsymbol{x}), & & \boldsymbol{x} \in \partial \Omega \cap \partial \Omega_{M} \\
\frac{\partial v_{M}(\boldsymbol{x})}{\partial \boldsymbol{n}_{M}} & =0, & & \boldsymbol{x} \in \Sigma:=\bar{\Omega}_{M} \cap \bar{\Omega}_{A},
\end{aligned}\right.
$$

( $\boldsymbol{n}_{E}$ denotes the outer unit normal vector for $\Omega_{E}$, where $\left.E \in\{M, A\}\right)$ and

$$
\left\{\begin{aligned}
\Delta v_{A}(\boldsymbol{x}) & =0, & & \boldsymbol{x} \in \Omega_{A} \\
v_{A}(\boldsymbol{x}) & =g(\boldsymbol{x}), & & \boldsymbol{x} \in \partial \Omega \cap \partial \Omega_{A} \\
\left.v_{A}\right|_{\Sigma} & =\left.v_{M}\right|_{\Sigma} . & &
\end{aligned}\right.
$$

Thus, $v_{M}$ and $v_{A}$ are harmonic functions in $\Omega_{M}$ and $\Omega_{A}$, respectively, and are independent of $K$.

REMARK 3.2. It should be noted that $v_{M}$ and therefore also $v_{A}$ are not necessarily uniquely defined by (3.2) and (3.3). For each path connected component of $\Omega_{M}$ whose boundary doesn't have an intersection with $\partial \Omega$ of non-zero measure $v_{M}$ is only determined up to a constant. This non-uniqueness, however, doesn't have any effect on the following derivations. If one wants to think of some unique choice for $v_{M}$ (and thus $v_{A}$ ) one may fix each of the arbitrary constants in some desirable way.

Now we introduce the function

$$
v(\boldsymbol{x})= \begin{cases}v_{M}(\boldsymbol{x}) & \text { for } \quad \boldsymbol{x} \in \Omega_{M}, \\ v_{A}(\boldsymbol{x}) & \text { for } \boldsymbol{x} \in \Omega_{A} .\end{cases}
$$

Because of the choice of the boundary conditions in (3.2) and (3.3) this function belongs to $H^{1}(\Omega)$. Then for any function $w \in H_{0}^{1}(\Omega)$ we have (see, e.g. [11, Corollary 2.6]):

$$
\begin{aligned}
\int_{\Omega} K \nabla v \cdot \nabla w d \boldsymbol{x} & =\sum_{E \in\{A, M\}} \int_{\Omega_{E}} K_{E} \nabla v_{E} \cdot \nabla w d \boldsymbol{x} \\
& =\sum_{E \in\{A, M\}} \int_{\partial \Omega_{E}} K_{E} \frac{\partial v_{E}}{\partial \boldsymbol{n}_{E}} w d S(\boldsymbol{x}) \quad\left(\text { since } v \text { is harmonic in } \Omega_{E}\right) \\
& =\delta \int_{\partial \Omega_{A}} \frac{\partial v_{A}}{\partial \boldsymbol{n}_{A}} w d S(\boldsymbol{x}), \quad\left(\text { since }\left.\nabla v_{M}(\boldsymbol{x}) \cdot \boldsymbol{n}_{M}\right|_{\Sigma}=0\right) .
\end{aligned}
$$

Since $u$ is the solution of (1.1), then for all $w \in H_{0}^{1}(\Omega)$ we have

$$
\int_{\Omega} K \nabla u \cdot \nabla w d \boldsymbol{x}=0
$$

Subtracting (3.6) from (3.5) yields

$$
\begin{aligned}
\int_{\Omega} K \nabla(v-u) \cdot \nabla w d \boldsymbol{x} & =\int_{\partial \Omega_{A}} \delta \frac{\partial v_{A}}{\partial \boldsymbol{n}_{A}} w d S(\boldsymbol{x}) \\
& \leq \delta\left\|\frac{\partial v_{A}}{\partial \boldsymbol{n}_{A}}\right\|_{H^{-\frac{1}{2}}\left(\partial \Omega_{A}\right)}\|w\|_{H^{\frac{1}{2}}\left(\partial \Omega_{A}\right)} \\
& \leq C \delta\|w\|_{H^{\frac{1}{2}}\left(\partial \Omega_{A}\right)},
\end{aligned}
$$

where we have used the Cauchy-Schwarz inequality and the fact, that $\left\|\frac{\partial v_{A}}{\partial \boldsymbol{n}_{A}}\right\|_{H^{-\frac{1}{2}}\left(\partial \Omega_{A}\right)}$ is independent of $\delta$. Choosing $w=v-u \in H_{0}^{1}(\Omega)$ and noting that $K(\boldsymbol{x}) \geq \delta$ in $\Omega$ we obtain

$$
\int_{\Omega} \delta \nabla(v-u) \cdot \nabla(v-u) d \boldsymbol{x} \leq C \delta\|v-u\|_{H^{\frac{1}{2}}\left(\partial \Omega_{A}\right)} .
$$


Using the trace theorem and the Poincaré inequality we deduce

$$
\int_{\Omega} \nabla(v-u) \cdot \nabla(v-u) d \boldsymbol{x} \leq C\|\nabla(v-u)\|_{L^{2}\left(\Omega_{A}\right)} \leq C\|\nabla(v-u)\|_{L^{2}(\Omega)} .
$$

Thus,

$$
\|\nabla(v-u)\|_{L^{2}(\Omega)} \leq C
$$

which implies (3.1), since $v$ is independent of $\delta$.

REMARK 3.3. The theory developed below concerns cell problems with boundary conditions as stated in (1.3). With some modifications it may also be used for cell problems with periodic boundary conditions, if the underlying $R E V$ is itself periodic.

The previous lemma plays a key role in the development of the further theory. For a better understanding of our strategy we first consider the special case that for each path-connected component of the highly conductive domain (i.e. $\Omega_{M}$ ) we have that its boundary has an intersection of non-zero measure with $\partial \Omega$. This special case is treated in the following subsection before we return to the general case in subsection 3.3. For a better understanding of the difference between the special and the general case we refer to Figure 3.1 .

3.2. A special case. The next proposition provides a means to approximate $\langle-K \nabla u\rangle_{\Omega}$. We do this by using the auxiliary function $v$ defined in the proof of Lemma 3.1. A crucial point here is the fact that $v$ can be found by solving two separate problems with constant coefficients.

PROPOSITION 3.4. Let the assumptions of Lemma 3.1 be satisfied. Additionally, we require that the boundary of each path-connected component of $\Omega_{M}$ has an intersection with $\partial \Omega$ of non-zero measure. Each path-connected component of $\Omega_{M}$ is furthermore assumed to be a finite union of domains each of which is star-shaped w.r.t. a ball. Finally, we let $v$ be defined by (3.2)-(3.4). (Note, that under our assumptions for $\Omega_{M}$ in the special case $v$ is actually uniquely defined by (3.2)-(3.4), cf. Remark 3.2.)

Then,

$$
\left|\langle K \nabla u\rangle_{\Omega}-\langle K \nabla v\rangle_{\Omega}\right|=\mathcal{O}(\delta), \quad \text { as } \delta \rightarrow 0 .
$$

Here and below $|\cdot|$ applied to elements from $\mathbb{R}^{n}$ denotes some norm.

Proof. By choosing $w=v-u \in H_{0}^{1}(\Omega)$ in (3.7) we obtain

$$
\begin{aligned}
\int_{\Omega} K \nabla(v-u) \cdot \nabla(v-u) d \boldsymbol{x} & \leq \delta C\|v-u\|_{H^{\frac{1}{2}}\left(\partial \Omega_{A}\right)} \\
& =\delta C\|v-u\|_{H^{\frac{1}{2}}\left(\partial \Omega_{M}\right)} \\
& \leq \delta C\|v-u\|_{H^{1}\left(\Omega_{M}\right)}
\end{aligned}
$$

by the trace theorem. Now, due to the properties of $\Omega_{M}$ we may apply Poincaré's inequality (cf. [8, Section 5.3]) and obtain

$$
\int_{\Omega_{M}} \nabla(v-u) \cdot \nabla(v-u) d \boldsymbol{x} \leq \delta C\|\nabla(v-u)\|_{L^{2}\left(\Omega_{M}\right)} .
$$

Thus,

$$
\|\nabla(v-u)\|_{L^{2}\left(\Omega_{M}\right)}=\mathcal{O}(\delta)
$$

and another application of Poincaré's inequality yields

$$
\|v-u\|_{H^{1}\left(\Omega_{M}\right)}=\mathcal{O}(\delta) .
$$

By the trace theorem we deduce that

$$
\|v-u\|_{H^{\frac{1}{2}}\left(\partial \Omega_{M}\right)}=\mathcal{O}(\delta) .
$$

Since $v-u$ is harmonic in $\Omega_{A}$ and has zero trace on $\partial \Omega \cap \partial \Omega_{A}$, and since $\partial \Omega_{A}=\left(\partial \Omega \cap \partial \Omega_{A}\right) \cup \Sigma$ we have by [11, Section 1.3] and (3.15) that

$$
\|v-u\|_{H^{1}\left(\Omega_{A}\right)} \leq C\|v-u\|_{H^{\frac{1}{2}}\left(\partial \Omega_{M}\right)}=\mathcal{O}(\delta) .
$$


From (3.14) and (3.16) it is straightforward to obtain (3.10).

Now, we are ready to state our main result for the special case under consideration.

THEOREM 3.5 (Main result - special case). Let the assumptions of Proposition 3.4 be satisfied and let $v$ be defined by (3.2)-(3.4). Then

$$
\left|\langle K \nabla u\rangle_{\Omega}-\frac{\left|\Omega_{M}\right|}{|\Omega|}\langle K \nabla v\rangle_{\Omega_{M}}\right|=\mathcal{O}(\delta), \quad \text { as } \delta \rightarrow 0 .
$$

REMARK 3.6. Before we proceed with the proof of Theorem 3.5 we note, that (3.17) combined with (1.4) provides a way to efficiently compute an approximation of the effective thermal conductivity tensor for high contrast media.

Proof. Obviously

$$
\left|\langle K \nabla u\rangle_{\Omega}-\frac{\left|\Omega_{M}\right|}{|\Omega|}\left\langle K \nabla v_{M}\right\rangle_{\Omega_{M}}\right| \leq \underbrace{\left|\langle K \nabla u\rangle_{\Omega}-\langle K \nabla v\rangle_{\Omega}\right|}_{=\mathcal{O}(\delta) \text { by Prop. 3.4 }}+\left|\langle K \nabla v\rangle_{\Omega}-\frac{\left|\Omega_{M}\right|}{|\Omega|}\langle\nabla v\rangle_{\Omega_{M}}\right|
$$

so it suffices to show

$$
\langle K \nabla v\rangle_{\Omega}-\frac{\left|\Omega_{M}\right|}{|\Omega|}\langle\nabla v\rangle_{\Omega_{M}}=\mathcal{O}(\delta)
$$

Observe, that

$$
\begin{aligned}
\langle K \nabla v\rangle_{\Omega} & =\frac{1}{|\Omega|}\left(\int_{\Omega_{M}} \nabla v d \boldsymbol{x}+\delta \int_{\Omega_{A}} \nabla v d \boldsymbol{x}\right) \\
& =\frac{1}{|\Omega|} \int_{\Omega_{M}} \nabla v d \boldsymbol{x}+\mathcal{O}(\delta) \quad \text { (since } v \text { is independent of } \delta \text { ) } \\
& =\frac{\left|\Omega_{M}\right|}{|\Omega|}\langle\nabla v\rangle_{\Omega_{M}}+\mathcal{O}(\delta) .
\end{aligned}
$$

Thus, (3.18) has been verified.

3.3. The general case. Now we return to the general case, where $\Omega_{M}$ may have path-connected components which are strictly inside $\Omega$. The following proposition applies Lemma 3.1 and essentially says that those path-connected components of the highly conductive subdomain may be neglected when approximating the effective thermal conductivity tensor $\tilde{K}$.

Proposition 3.7. Assume the same setting as in the statement of Lemma 3.1. Additionally, let $\widetilde{\Omega}_{M}$ be the union of path-connected components of $\Omega_{M}$ that do not touch the boundary $\partial \Omega$, i.e. $\left|\partial \widetilde{\Omega}_{M} \cap \partial \Omega\right|=0$. Then

$$
\left|\langle K \nabla u\rangle_{\Omega}-\frac{\left|\Omega^{*}\right|}{|\Omega|}\langle K \nabla u\rangle_{\Omega^{*}}\right|=\mathcal{O}(\delta) \text {, as } \delta \rightarrow 0
$$

where $\Omega^{*}:=$ interior $\left(\Omega \backslash \widetilde{\Omega}_{M}\right)$. (See Figure 3.1 for a visual presentation of various components of $\Omega$ ).

Proof. It is easy to see that

$$
\left|\langle K \nabla u\rangle_{\Omega}-\frac{\left|\Omega^{*}\right|}{|\Omega|}\langle K \nabla u\rangle_{\Omega^{*}}\right|=\frac{1}{|\Omega|}\left|\int_{\widetilde{\Omega}_{M}} K \nabla u d \boldsymbol{x}\right| .
$$

Define $\widetilde{v}_{M}:=\left.u\right|_{\widetilde{\Omega}_{M}}$, where $u$ solves (1.1). Clearly, by its definition $\widetilde{v}_{M}$ satisfies the b.v.p.

$$
\left\{\begin{array}{l}
\Delta \widetilde{v}_{M}=0, \text { in } \widetilde{\Omega}_{M} \\
\frac{\partial \widetilde{v}_{M}}{\partial \boldsymbol{n}_{M}}=\delta \frac{\partial u_{A}}{\partial \boldsymbol{n}_{M}} \quad \text { on } \partial \widetilde{\Omega}_{M}
\end{array}\right.
$$

where $u_{A}=\left.u\right|_{\Omega_{A}}$. Obviously, we have that

$$
\left|\int_{\widetilde{\Omega}_{M}} K \nabla u d \boldsymbol{x}\right| \leq\|K \nabla u\|_{L^{1}\left(\widetilde{\Omega}_{M}\right)} \leq C\|K \nabla u\|_{L^{2}\left(\widetilde{\Omega}_{M}\right)}=C\left\|\nabla \widetilde{v}_{M}\right\|_{L^{2}\left(\widetilde{\Omega}_{M}\right)} .
$$


By [11, Section 1.4] we know, that

$$
\left\|\nabla \widetilde{v}_{M}\right\|_{L^{2}\left(\widetilde{\Omega}_{M}\right)} \leq C \delta\left\|\frac{\partial u_{A}}{\partial \boldsymbol{n}_{M}}\right\|_{H^{-\frac{1}{2}}\left(\partial \widetilde{\Omega}_{M}\right)},
$$

from where we deduce by [11, Theorem 1.6] that

$$
\left\|\nabla \widetilde{v}_{M}\right\|_{L^{2}\left(\widetilde{\Omega}_{M}\right)} \leq C \delta\|u\|_{H^{1}(\Omega)}
$$

Thus, we have that

$$
\left\|\nabla \widetilde{v}_{M}\right\|_{L^{2}\left(\widetilde{\Omega}_{M}\right)} \leq C \delta\left(\|u\|_{L^{2}(\Omega)}+\|\nabla u\|_{L^{2}(\Omega)}\right)
$$

and it suffices to show, that $\|u\|_{L^{2}(\Omega)}$ and $\|\nabla u\|_{L^{2}(\Omega)}$ are bounded independently of $\delta$.

Lemma 3.1 yields that $\|\nabla u\|_{L^{2}(\Omega)}$ is bounded independently of $\delta$. To make an estimate for $\|u\|_{L^{2}(\Omega)}$ we use the following construction. Let $v^{g}$ be the harmonic extension of $g$ from (1.1). By [11, Section 1.3] we have that

$$
\left\|v^{g}\right\|_{H^{1}(\Omega)} \leq C\|g\|_{H^{\frac{1}{2}}(\partial \Omega)}
$$

where, as usual, $C$ is a constant independent of $\delta$.

By Poincaré's inequality we have for $u-v^{g} \in H_{0}^{1}(\Omega)$

$$
\left\|u-v^{g}\right\|_{L^{2}(\Omega)} \leq\|\nabla u\|_{L^{2}(\Omega)}+\left\|\nabla v^{g}\right\|_{L^{2}(\Omega)} \leq C,
$$

where we have used Lemma 3.1 and the fact that $v^{g}$ is independent of $\delta$. (3.24) combined with (3.25) yields the uniform (w.r.t. $\delta$ ) boundedness of $\|u\|_{L^{2}(\Omega)}$, which concludes the proof.

REMARK 3.8. Observe, that by the proof of Proposition 3.7 we have in particular that the solution $u$ of (1.1) is bounded independently of $\delta$ in $H^{1}$-norm.

The next proposition provides a means to approximate $\langle K \nabla u\rangle_{\Omega^{*}}$, which in turn according to the previous statement is sufficient to approximate the effective thermal conductivity tensor of the entire domain. We do this by constructing a function similarly to the auxiliary one in Lemma 3.1. Again, the crucial aspect to observe here is that the restriction of this function to $\Omega_{M}$ and $\Omega_{A}$, respectively, solves a constant coefficient problem.

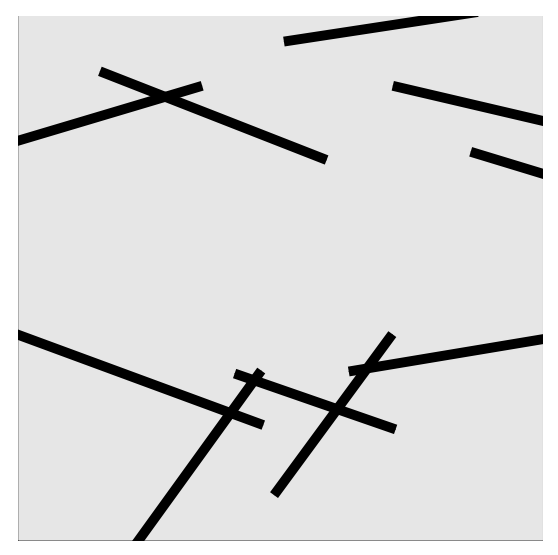

(a) Setting in the special case, where all path-connected components of $\Omega_{M}$ touch the boundary of $\Omega$.

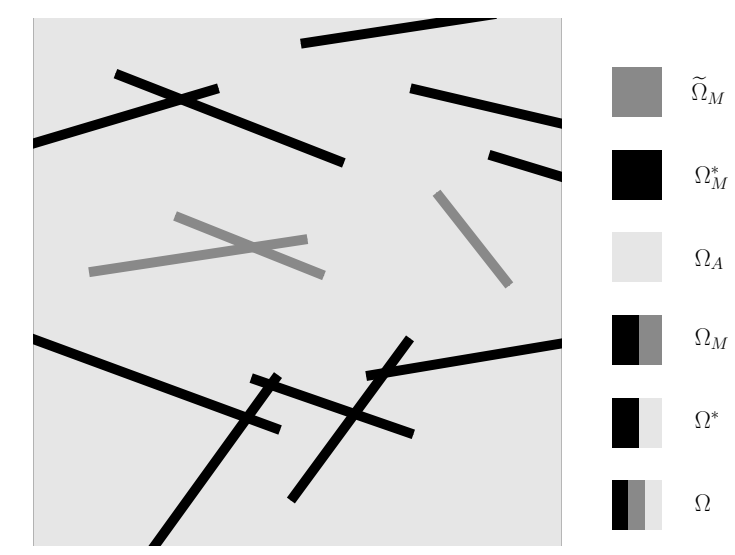

(b) Setting in the general case, where path-connected components of $\Omega_{M}$ may not touch the boundary of $\Omega$. 
Proposition 3.9. Let the assumptions of Lemma 3.1 be satisfied. Additionally, let $\Omega^{*}$ be defined as in Proposition 3.7 and its proof. Furthermore, let

$$
v^{*}=\left\{\begin{array}{l}
v_{M}^{*}, \quad \text { in } \Omega_{M}^{*} \\
u, \quad \text { in } \widetilde{\Omega}_{M} \\
v_{A}^{*}, \quad \text { in } \Omega_{A}
\end{array}\right.
$$

where $v_{M}^{*}$ and $v_{A}^{*}$ are defined by

$$
\left\{\begin{array}{rll}
\Delta v_{M}^{*}=0 & \text { in } \Omega_{M}^{*} \\
v_{M}^{*}=g & \text { on } \partial \Omega \cap \partial \Omega_{M}^{*} \\
\frac{\partial v_{M}^{*}}{\partial \boldsymbol{n}_{M}}=0 & \text { on } \Sigma^{*}
\end{array}\right.
$$

and

$$
\left\{\begin{array}{rlrl}
\Delta v_{A}^{*}=0 & & \text { in } \Omega_{A} \\
v_{A}^{*}=g & & \text { on } \partial \Omega \cap \partial \Omega_{A} \\
v_{A}^{*} & =v_{M}^{*} & & \text { on } \Sigma^{*} \\
v_{A}^{*} & =u & & \text { on } \Sigma \backslash \Sigma^{*}=\partial \widetilde{\Omega}_{M},
\end{array}\right.
$$

where $\Sigma^{*}:=\partial \Omega_{M}^{*} \cap \Sigma$ and $\Omega_{M}^{*}=$ interior $\left(\Omega^{*} \backslash \Omega_{A}\right)$. Also, we require that each path-connected component of $\Omega_{M}^{*}$ is a finite union of domains each of which is star-shaped w.r.t. a ball. (Note, that we already know, that by construction each path-connected component of $\Omega_{M}^{*}$ touches $\partial \Omega$.)

Then,

$$
\left|\langle K \nabla u\rangle_{\Omega^{*}}-\left\langle K \nabla v^{*}\right\rangle_{\Omega^{*}}\right|=\mathcal{O}(\delta), \quad \text { as } \delta \rightarrow 0
$$

Proof. Let $w \in H_{0}^{1}\left(\Omega^{*}\right)$. Then similarly to (3.5) in Lemma 3.1 we obtain

$$
\int_{\Omega^{*}} K \nabla v^{*} \cdot \nabla w d \boldsymbol{x}=\int_{\partial \Omega_{A}} \delta \frac{\partial v_{A}^{*}}{\partial \boldsymbol{n}_{A}} w d S(\boldsymbol{x}) .
$$

Clearly, we have

$$
\int_{\Omega^{*}} K \nabla u \cdot \nabla w d \boldsymbol{x}=0
$$

and subtracting (3.31) from (3.30) yields

$$
\int_{\Omega^{*}} K \nabla\left(v^{*}-u\right) \cdot \nabla w d \boldsymbol{x}=\delta \int_{\partial \Omega_{A}} \frac{\partial v_{A}^{*}}{\partial \boldsymbol{n}_{A}} w d S(\boldsymbol{x}) \leq \delta\left\|\frac{\partial v_{A}^{*}}{\partial \boldsymbol{n}_{A}}\right\|_{H^{-\frac{1}{2}}\left(\partial \Omega_{A}\right)}\|w\|_{H^{\frac{1}{2}}\left(\partial \Omega_{A}\right)} .
$$

By Remark 3.8 we know that $\|u\|_{H^{1}(\Omega)} \leq C$, where $C$ as throughout this paper does not depend on $\delta$. Therefore,

$$
\left\|v_{A}^{*}\right\|_{H^{\frac{1}{2}}\left(\partial \widetilde{\Omega}_{M}\right)}=\|u\|_{H^{\frac{1}{2}}\left(\partial \widetilde{\Omega}_{M}\right)} \leq C
$$

by the trace theorem. Thus,

$$
\begin{aligned}
\left\|v_{A}^{*}\right\|_{H^{1}\left(\Omega_{A}\right)} & \leq C\left\|v_{A}^{*}\right\|_{H^{\frac{1}{2}}\left(\partial \Omega_{A}\right)} \\
& \leq C\left(\left\|v_{A}^{*}\right\|_{H^{\frac{1}{2}}\left(\partial \widetilde{\Omega}_{M}\right)}+\left\|v_{A}^{*}\right\|_{H^{\frac{1}{2}\left(\Sigma^{*}\right)}}+\left\|v_{A}^{*}\right\|_{H^{\frac{1}{2}}\left(\partial \Omega_{A} \cap \partial \Omega\right)}\right) \\
& =C\left(\left\|v_{A}^{*}\right\|_{H^{\frac{1}{2}}\left(\partial \widetilde{\Omega}_{M}\right)}+\left\|v_{M}^{*}\right\|_{H^{\frac{1}{2}\left(\Sigma^{*}\right)}}+\|g\|_{H^{\frac{1}{2}}\left(\partial \Omega_{A} \cap \partial \Omega\right)}\right) \\
& \leq C,
\end{aligned}
$$


where we have used [11, Section 1.3], (3.33), and the fact that $\left.v_{M}^{*}\right|_{\Omega_{M}^{*}}$ does not depend on $\delta$ by (3.27).

Therefore, by [11, Theorem 1.6] we obtain

$$
\left\|\frac{\partial v_{A}^{*}}{\partial \boldsymbol{n}_{A}}\right\|_{H^{-\frac{1}{2}}\left(\partial \Omega_{A}\right)} \leq C
$$

Combining this with (3.32) we are left with

$$
\int_{\Omega^{*}} K \nabla\left(v^{*}-u\right) \cdot \nabla w d \boldsymbol{x} \leq \delta C\|w\|_{H^{\frac{1}{2}}\left(\partial \Omega_{A}\right)} .
$$

Choosing $w=v^{*}-u \in H_{0}^{1}\left(\Omega^{*}\right)$ we obtain

$$
\int_{\Omega^{*}} K \nabla\left(v^{*}-u\right) \cdot \nabla\left(v^{*}-u\right) d \boldsymbol{x} \leq \delta C\left\|v^{*}-u\right\|_{H^{\frac{1}{2}}\left(\partial \Omega_{A}\right)} \leq \delta C\left\|v^{*}-u\right\|_{H^{1}\left(\Omega_{M}^{*}\right)}
$$

by the trace theorem. Now, due to the properties of $\Omega_{M}^{*}$ we may apply Poincaré's inequality (cf. [8, Section 5.3]) and obtain

$$
\int_{\Omega_{M}^{*}} \nabla\left(v^{*}-u\right) \cdot \nabla\left(v^{*}-u\right) d \boldsymbol{x} \leq \delta C\left\|\nabla\left(v^{*}-u\right)\right\|_{L^{2}\left(\Omega_{M}^{*}\right)} .
$$

Thus, another application of Poincaré's inequality yields

$$
\left\|v^{*}-u\right\|_{H^{1}\left(\Omega_{M}^{*}\right)}=\mathcal{O}(\delta) .
$$

By the trace theorem we deduce that

$$
\left\|v^{*}-u\right\|_{H^{\frac{1}{2}}\left(\partial \Omega_{M}^{*}\right)}=\mathcal{O}(\delta) .
$$

Since $v^{*}-u$ is harmonic in $\Omega_{A}$ and has zero trace on $\left(\partial \Omega \cap \partial \Omega_{A}\right) \cup\left(\Sigma \backslash \Sigma^{*}\right)$, and since $\partial \Omega_{A}=\left(\partial \Omega \cap \partial \Omega_{A}\right) \cup$ $\left(\Sigma \backslash \Sigma^{*}\right) \cup \Sigma^{*}$ we have by [11, Section 1.3] and (3.39)

$$
\left\|v^{*}-u\right\|_{H^{1}\left(\Omega_{A}\right)} \leq C\left\|v^{*}-u\right\|_{H^{\frac{1}{2}}\left(\partial \Omega_{M}^{*}\right)}=\mathcal{O}(\delta) .
$$

From (3.38) and (3.40) it is straightforward to obtain (3.29)

We are now ready to state our main result:

THEOREM 3.10 (Main result - general case). Let the assumptions of Lemma 3.1 be satisfied. Furthermore, let $\Omega^{*}, \Sigma^{*}$, and $\Omega_{M}^{*}$ be defined as in Propositions 3.7 and 3.9, respectively. As in the previous proposition we also have to assume that each path-connected component of $\Omega_{M}^{*}$ is a finite union of domains each of which is star-shaped w.r.t. a ball. Then, if $v_{M}^{*}$ is the unique solution of (3.27) we have that

$$
\left|\langle K \nabla u\rangle_{\Omega}-\frac{\left|\Omega_{M}^{*}\right|}{|\Omega|}\left\langle K \nabla v_{M}^{*}\right\rangle_{\Omega_{M}^{*}}\right|=\mathcal{O}(\delta), \quad \text { as } \delta \rightarrow 0,
$$

REMARK 3.11. Analogous to Remark 3.6, it should again be noted that estimate (3.41) is meaningful only if $\left|\Omega_{M}^{*}\right| \neq 0$.

Proof. Let $v^{*}$ be defined by (3.26)-(3.28). We obviously have

$$
\begin{aligned}
\left|\langle K \nabla u\rangle_{\Omega}-\frac{\left|\Omega_{M}^{*}\right|}{|\Omega|}\left\langle K \nabla v^{*}\right\rangle_{\Omega_{M}^{*}}\right| \leq & \underbrace{\left|\langle K \nabla u\rangle_{\Omega}-\frac{\left|\Omega^{*}\right|}{|\Omega|}\langle K \nabla u\rangle_{\Omega^{*}}\right|}_{=\mathcal{O}(\delta) \text { by Prop. 3.7 }}+\underbrace{\frac{\left|\Omega^{*}\right|}{|\Omega|}\left|\langle K \nabla u\rangle_{\Omega^{*}}-\left\langle K \nabla v^{*}\right\rangle_{\Omega^{*}}\right|}_{=\mathcal{O}(\delta) \text { by Prop. 3.9 }} \\
& +\left|\frac{\left|\Omega^{*}\right|}{|\Omega|}\left\langle K \nabla v^{*}\right\rangle_{\Omega^{*}}-\frac{\left|\Omega_{M}^{*}\right|}{|\Omega|}\left\langle K \nabla v^{*}\right\rangle_{\Omega_{M}^{*}}\right| .
\end{aligned}
$$

Therefore, it suffices to show

$$
\frac{\left|\Omega^{*}\right|}{|\Omega|}\left\langle K \nabla v^{*}\right\rangle_{\Omega^{*}}-\frac{\left|\Omega_{M}^{*}\right|}{|\Omega|}\left\langle K \nabla v^{*}\right\rangle_{\Omega_{M}^{*}}=\mathcal{O}(\delta) .
$$


Observe, that

$$
\begin{aligned}
-\frac{\left|\Omega^{*}\right|}{|\Omega|}\left\langle K \nabla v^{*}\right\rangle_{\Omega^{*}} & =\frac{-1}{|\Omega|}\left(\int_{\Omega_{M}^{*}} \nabla v^{*} d \boldsymbol{x}+\int_{\Omega_{A}} \delta \nabla v^{*} d \boldsymbol{x}\right) \\
& =\frac{-1}{|\Omega|} \int_{\Omega_{M}^{*}} \nabla v^{*} d \boldsymbol{x}+\mathcal{O}(\delta), \quad \text { by (3.34) } \\
& =-\frac{\left|\Omega_{M}^{*}\right|}{|\Omega|}\left\langle K \nabla v^{*}\right\rangle_{\Omega_{M}^{*}}+\mathcal{O}(\delta),
\end{aligned}
$$

which yields (3.42).

4. A $\delta$-independent algorithm for high contrast materials. Theorem 3.10 and Remark 3.6 , provide the theoretical justification of an algorithm, which can be used to compute an approximation of the effective thermal conductivity tensor of an REV $\Omega$ consisting of a highly conductive $\left(\Omega_{M}\right)$ and a lowly conductive $\left(\Omega_{A}\right)$ part, where the conductivity $K_{M}$ in $\Omega_{M}$ is much larger than the conductivity $K_{A}$ in $\Omega_{A}$. The computational cost of this algorithm is independent of $\delta$. As stated above, we are typically interested in those materials for which $\left|\Omega_{M}\right|$ is significantly smaller than $\left|\Omega_{A}\right|$. Since with our approach the only problem that needs to be solved is a discretization of (3.27) we can expect to have substantially reduced memory requirements compared with standard algorithms solving a discretization of the full problem, i.e. (1.3), whenever $\left|\Omega_{M}\right| \ll\left|\Omega_{A}\right|$.

Note, that due to equation (3.43) we see that the flux in $\Omega_{A}$ is $\mathcal{O}(\delta)$ and may, therefore, be asymptotically neglected as $\delta \rightarrow 0$. By the proof of Proposition 3.7 we know that the same is true for the flux in $\widetilde{\Omega}_{M}$. Nevertheless, instead of disregarding $\Omega_{A}$ and $\Omega_{M}$ completely in the calculation of the effective thermal conductivity tensor, we may of course prescribe some constant temperature gradient in $\Omega_{A}$ and a temperature gradient being $\mathcal{O}(\delta)$ in $\widetilde{\Omega}_{M}$. Certainly, for $\delta \rightarrow 0$ the resulting fluxes tend to zero, as well. Nonetheless, for a specific choice for $\delta$ we may still hope to (and in many numerically tested cases do) obtain better estimates of the effective thermal conductivity tensors. In the numerical examples presented in section 5 the temperature in $\Omega_{A}$ is approximated by linearly interpolating the (Dirichlet) boundary conditions in (1.3), leading to a constant approximation of the temperature gradient. The temperature gradient in $\widetilde{\Omega}_{M}$ is obtained in the same way and then scaled by $\delta$. Note that, as mentioned in section 2 , the objective to capture the influence of $\Omega_{A}$ was previously discussed in [6]. Unlike in [6], however, we don't solve additional problems in $\Omega_{A}$, which makes our taking into account contributions of $\Omega_{A}$ significantly cheaper.

Based on our considerations above, we may now formulate Algorithm 1 for computing an approximation $\tilde{K}^{C O+A}$ of $\tilde{K}$ for high contrast REV's (here ${ }^{C O+A}$ stands for “conductive only plus air").

REMARK 4.1. The simplified formula (4.2) provides an efficient method for upscaling materials of high contrast. In the case of fibrous materials with fibers that have a high ratio between their lengths and diameters there is a possibility for a further simplification of the model and for a substantial reduction of the complexity. In this case we can model the fibers as one dimensional bodies that form trust-like structures with thermal conductivities depending on the physical conductivities of the fibers and their diameters. The algorithm based on this model was described and analyzed in [14]. The numerical experiments in [14] show a reduction of computational resources, i.e. memory and CPU-time, of several orders of magnitude.

5. Numerical Experiments and Conclusions. We now test Algorithm 1 on two fiber geometries and one foam geometry with a sequence of increasing contrasts, i.e. decreasing $\delta$. The geometries shown in Figures 5.1(a), 5.2(a), and 5.3(a), respectively, were generated and plotted with the GeoDict2007 software.

The two fiber structures are cubic and have a solid volume fraction of 5\% (Figure 5.1(a)) and 30\% (Figure 5.2(a)), respectively. $80 \%$ of the fiber volume is occupied by thin fibers (colored light gray), whereas the remaining $20 \%$ are taken up by thick fibers (colored dark gray). Both geometries are discretized by $200^{3}$ voxels. The cubic foam geometry (Figure 5.3(a)) has a solid volume fraction of $8.75 \%$, and it is discretized by $100^{3}$ voxels.

In this context we would once again like to point out, that we do not make any statement concerning the question whether the structures shown in Figures 5.1(a)-5.3(a) constitute REVs. In particular we do not claim, that (physically meaningful) effective thermal conductivity tensors exist for all three structures 

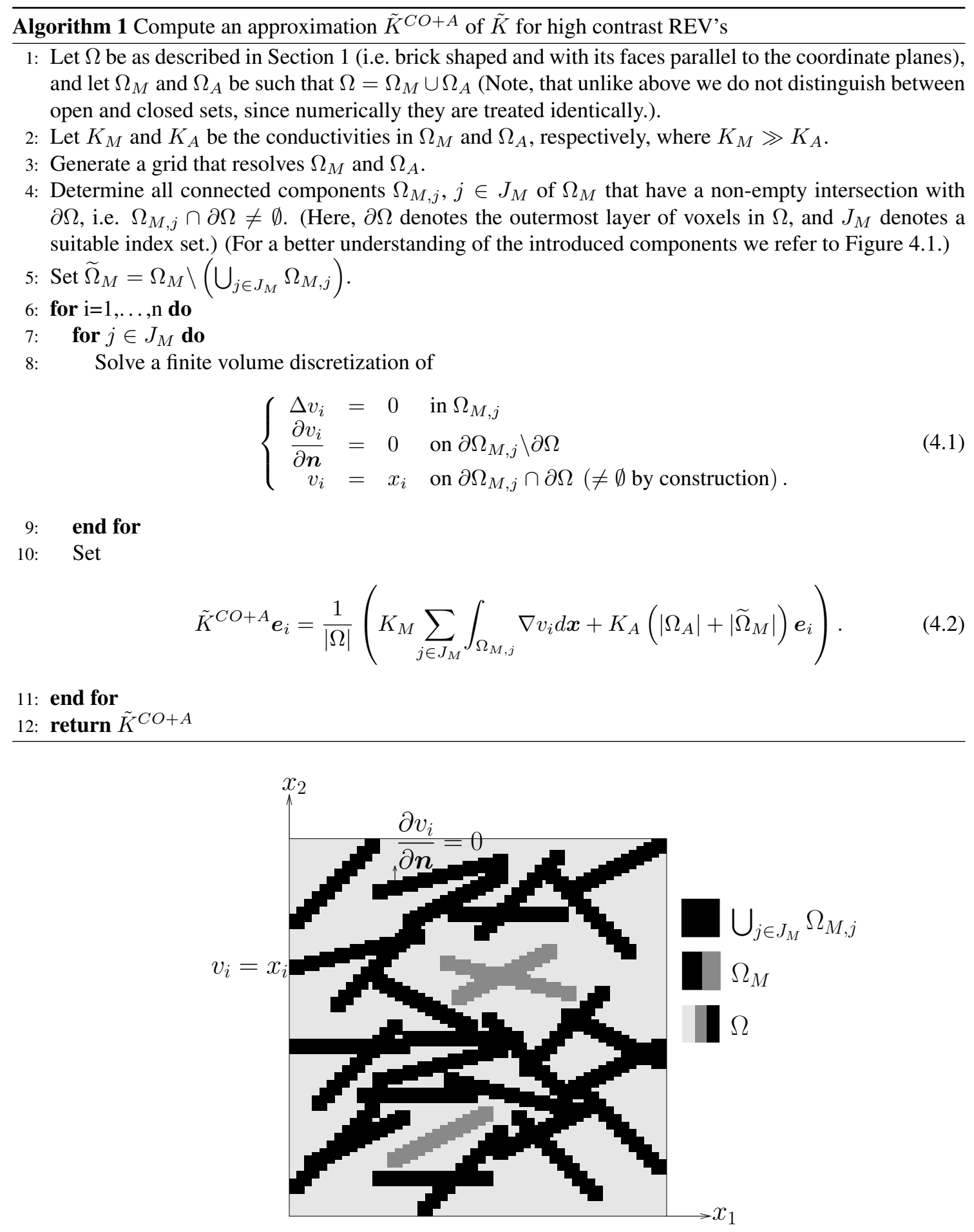

FIG. 4.1. Voxelized approximation of $\Omega$ and its components.

and all considered contrasts. Certainly, the main application of Algorithm 1 is to compute effective thermal conductivity tensors. When they exist, their approximation via Algorithm 1 is very much preferable over computations on the whole domain due to savings in memory and a contrast independent condition number of the resulting linear system. Nevertheless, the statement, that (3.41) holds is independent of the question, whether the configuration under consideration, i.e. geometry and contrast, admits the notion of an effective thermal conductivity tensor.

For each geometry and each $\delta$ we consider three cell problems with boundary conditions as in (1.3). 
Each boundary value problem is then solved by a standard finite volume discretization on the full domain (yielding $\tilde{K}_{i}:=\tilde{K} \boldsymbol{e}_{i}, i=1,2,3$ ). We then compare these reference results to the outputs given by Algorithm 1 (yielding $\left.\tilde{K}^{C O+A}=\left(\tilde{K}_{i, j}^{C O+A}\right)_{i, j=1, \ldots, 3}\right)$. Note, that our main result (3.41) unlike (4.2) doesn't involve any consideration of the heat flux in $\Omega_{A}$ and $\widetilde{\Omega}_{M}$. Therefore, to also specifically test the statement of Theorem 3.10 we define

$$
\tilde{K}^{C O} \boldsymbol{e}_{i}=\frac{1}{|\Omega|} K_{M} \sum_{j \in J_{M}} \int_{\Omega_{M, j}} \nabla v_{i} d \boldsymbol{x}
$$

corresponding to (4.2) without the $\mathcal{O}(\delta)$ terms.

To verify (3.41) and (4.2) we report

$$
\max _{i, j=1, \ldots, n}\left|\tilde{K}_{i, j}-\tilde{K}_{i, j}^{C O+A}\right| \quad \text { and } \max _{i, j=1, \ldots, n}\left|\tilde{K}_{i, j}-\tilde{K}_{i, j}^{C O}\right| .
$$

In Tables 5.1-5.3 we report the computed tensors for some representative contrasts. Figures 5.1(b)5.3(b) display the results for the different geometries, respectively. As we can see the quantities stated in (5.2) decay essentially linearly with $\delta$, which is in coherence with what we have shown above and also suggests the optimality of our estimates. It should furthermore be noted, that the constant implicitly involved in estimate (3.41) appears to be rather small. From a practical point of view, this is certainly crucial. Of course, the constant is very much geometry dependent, but we can see, that for our generated structures, which are at least somewhat representative for a class of industrial problems, we obtain satisfactory results even if the contrast is only $1: 10$.

Also we would like to point out, that the additional $\mathcal{O}(\delta)$ terms in (4.2) significantly improve the approximation of $\tilde{K}$ compared to formula (5.1), which doesn't involve these extra summands. The improvement seems to be more pronounced for geometries with high porosity, which is not surprising, since in these geometries the contribution of the air to the overall heat conduction is relatively bigger.

In addition to the absolute error terms given by (5.2) we also report two corresponding quantities assessing the relative error of the method. For this we consider the following error terms, where we have used a scaling by the diagonal entries of $\tilde{K}$

$$
\max _{i, j=1, \ldots, n}\left|\frac{\tilde{K}_{i, j}-\tilde{K}_{i, j}^{C O+A}}{\tilde{K}_{i, i}}\right| \quad \text { and } \max _{i, j=1, \ldots, n}\left|\frac{\tilde{K}_{i, j}-\tilde{K}_{i, j}^{C O}}{\tilde{K}_{i, i}}\right| .
$$

The developed theory justifies our algorithm in an asymptotic sense, i.e. it is valid for sufficiently small $\delta$. Nevertheless, even for contrasts of $1: 10$ (i.e. delta $=0.1$ ) the relative error according to (5.3) is about $10 \%$ for all considered in the paper geometries. For many applications this is already quite a satisfactory result. Further, as $\delta$ decreases the relative error is diminished. Comparing the Tables 5.1(a) and 5.1(b) we see that the error in computing $\tilde{K}^{C O}$ and $\tilde{K}^{C O+A}$ is about $43 \%$ and $5 \%$, for $\delta=0.01$ and $7 \%$ and less that $1 \%$, for $\delta=0.001$. This indicates that taking into account the $\mathcal{O}(\delta)$ terms in (4.2) improves our estimates. However, in the case of materials with foam structure this effect is less profound, namely, $20 \%$ and $1.4 \%$ for $\delta=0.01$, as seen from Table 5.3. In all cases the error diminishes linearly as $\delta \rightarrow 0$.

Acknowledgments. This research was performed during the meetings of the authors in Kaiserslautern, Germany, Sozopol, Bulgaria and College Station, Texas. The preliminary version of the results were summarized in the ITWM Technical Report [10] completed in November 2007. The current paper is an improved version of the text of [10] and significantly updated section with the numerical experiments and was completed while R. Lazarov was visiting the University of Kaiserslautern in July 2008 as a scholar of the European School for Industrial Mathematics (ESIM), sponsored by the Erasmus Mundus program of the EU.

The research of R. Lazarov was supported in parts by NSF Grants DMS-0713829, CNS-ITR-0540136, and by award KUS-C1-016-04, made by King Abdullah University of Science and Technology (KAUST). O. Iliev was supported by DAAD-PPP D/07/10578, I. Rybak was supported by INTAS-30-50-4355 project and by DAAD-PPP A/05/57218 grant, and J. Willems was supported by DAAD-PPP D/07/10578 and the German National Academic Foundation. 


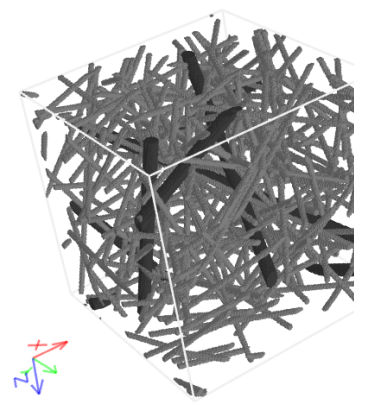

(a) Structure with 5\% fiber content.

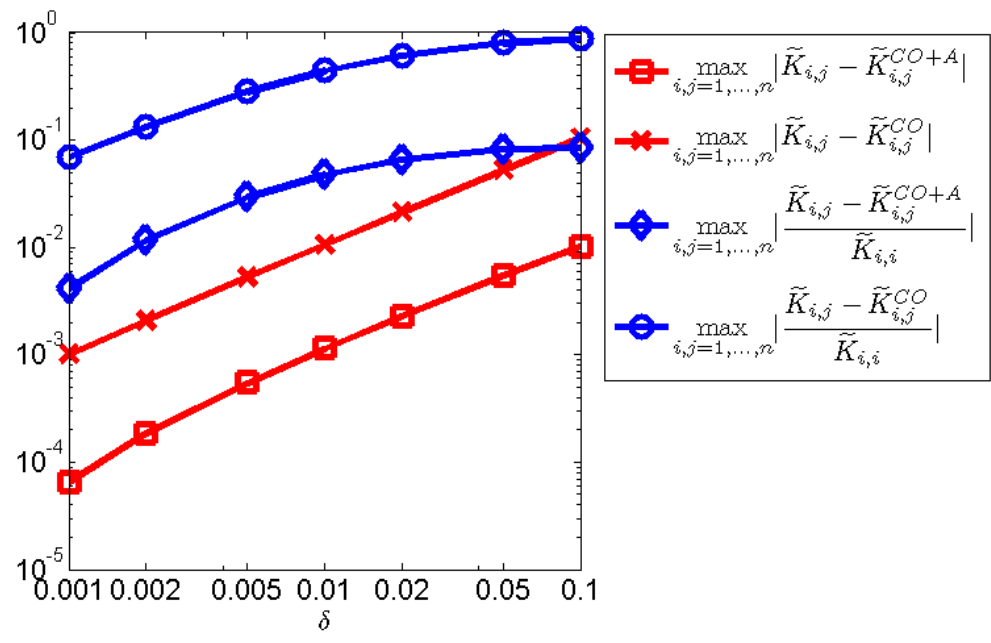

(b) Errors for different contrasts.

FIG. 5.1. Performance of Algorithm 1 for a sparse fiber geometry.

(a) $\delta=0.01$

\begin{tabular}{|ccc|ccc|ccc|}
\hline \multicolumn{3}{|c|}{$\tilde{K}$} & \multicolumn{3}{c|}{$\tilde{K}^{C O}$} & \multicolumn{3}{c|}{$\tilde{K}^{C O+A}$} \\
\hline $2.40 \mathrm{e}-02$ & $4.13 \mathrm{e}-04$ & $2.96 \mathrm{e}-05$ & $1.34 \mathrm{e}-02$ & $4.15 \mathrm{e}-04$ & $3.10 \mathrm{e}-05$ & $2.29 \mathrm{e}-02$ & $4.15 \mathrm{e}-04$ & $3.10 \mathrm{e}-05$ \\
$4.13 \mathrm{e}-04$ & $2.64 \mathrm{e}-02$ & $3.67 \mathrm{e}-04$ & $4.23 \mathrm{e}-04$ & $1.58 \mathrm{e}-02$ & $3.84 \mathrm{e}-04$ & $4.23 \mathrm{e}-04$ & $2.53 \mathrm{e}-02$ & $3.84 \mathrm{e}-04$ \\
$2.96 \mathrm{e}-05$ & $3.67 \mathrm{e}-04$ & $2.55 \mathrm{e}-02$ & $2.89 \mathrm{e}-05$ & $3.80 \mathrm{e}-04$ & $1.49 \mathrm{e}-02$ & $2.89 \mathrm{e}-05$ & $3.80 \mathrm{e}-04$ & $2.44 \mathrm{e}-02$ \\
\hline
\end{tabular}

(b) $\delta=0.001$.

\begin{tabular}{|ccc|ccc|ccc|}
\hline \multicolumn{3}{|c|}{$\tilde{K}$} & \multicolumn{3}{c|}{$\tilde{K}^{C O}$} & \multicolumn{3}{c|}{$\tilde{K}^{C O+A}$} \\
\hline $1.44 \mathrm{e}-02$ & $4.18 \mathrm{e}-04$ & $2.98 \mathrm{e}-05$ & $1.34 \mathrm{e}-02$ & $4.15 \mathrm{e}-04$ & $3.10 \mathrm{e}-05$ & $1.43 \mathrm{e}-02$ & $4.15 \mathrm{e}-04$ & $3.10 \mathrm{e}-05$ \\
$4.18 \mathrm{e}-04$ & $1.68 \mathrm{e}-02$ & $3.79 \mathrm{e}-04$ & $4.23 \mathrm{e}-04$ & $1.58 \mathrm{e}-02$ & $3.84 \mathrm{e}-04$ & $4.23 \mathrm{e}-04$ & $1.67 \mathrm{e}-02$ & $3.84 \mathrm{e}-04$ \\
$2.98 \mathrm{e}-05$ & $3.79 \mathrm{e}-04$ & $1.59 \mathrm{e}-02$ & $2.89 \mathrm{e}-05$ & $3.80 \mathrm{e}-04$ & $1.49 \mathrm{e}-02$ & $2.89 \mathrm{e}-05$ & $3.80 \mathrm{e}-04$ & $1.59 \mathrm{e}-02$ \\
\hline
\end{tabular}

TABLE 5.1

Effective thermal conductivity tensors for the fiber geometry of Figure 5.1(a) for different contrasts.

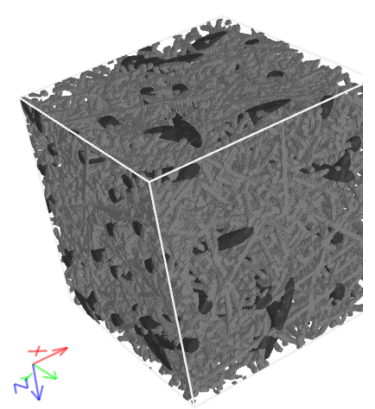

(a) Structure with $30 \%$ fiber content.

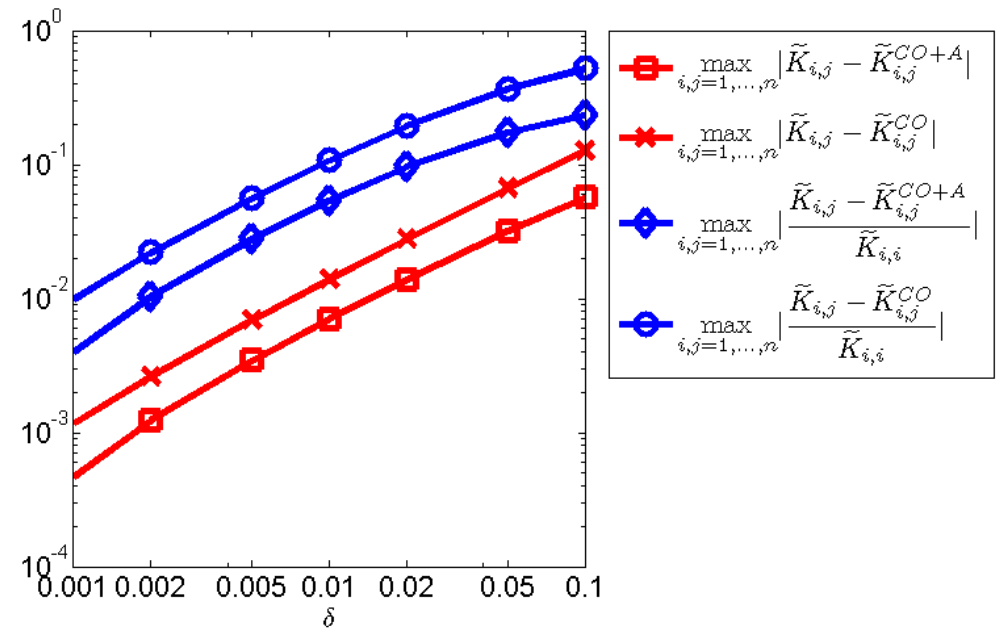

(b) Errors for different contrasts.

FIG. 5.2. Performance of Algorithm 1 for a dense fiber geometry. 


\begin{tabular}{|ccc|ccc|ccc|}
\hline \multicolumn{3}{|c|}{$\tilde{K}$} & \multicolumn{3}{c|}{$\tilde{K}^{C O}$} & \multicolumn{3}{c|}{$\tilde{K}^{C O+A}$} \\
\hline $1.33 \mathrm{e}-01$ & $2.18 \mathrm{e}-03$ & $1.95 \mathrm{e}-03$ & $1.19 \mathrm{e}-01$ & $2.31 \mathrm{e}-03$ & $2.08 \mathrm{e}-03$ & $1.26 \mathrm{e}-01$ & $2.31 \mathrm{e}-03$ & $2.08 \mathrm{e}-03$ \\
$2.18 \mathrm{e}-03$ & $1.31 \mathrm{e}-01$ & $8.52 \mathrm{e}-04$ & $2.31 \mathrm{e}-03$ & $1.16 \mathrm{e}-01$ & $8.98 \mathrm{e}-04$ & $2.31 \mathrm{e}-03$ & $1.23 \mathrm{e}-01$ & $8.98 \mathrm{e}-04$ \\
$1.95 \mathrm{e}-03$ & $8.52 \mathrm{e}-04$ & $1.30 \mathrm{e}-01$ & $2.07 \mathrm{e}-03$ & $9.02 \mathrm{e}-04$ & $1.16 \mathrm{e}-01$ & $2.07 \mathrm{e}-03$ & $9.02 \mathrm{e}-04$ & $1.23 \mathrm{e}-01$ \\
\hline
\end{tabular}

TABLE 5.2

Effective thermal conductivity tensors for the fiber geometry of Figure 5.2(a) for $\delta=0.01$.

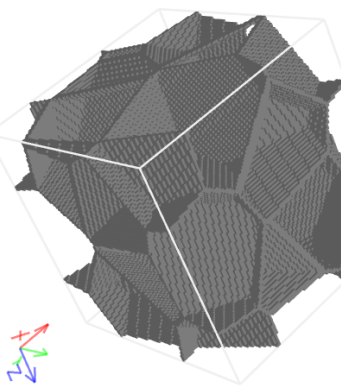

(a) Foam structure.

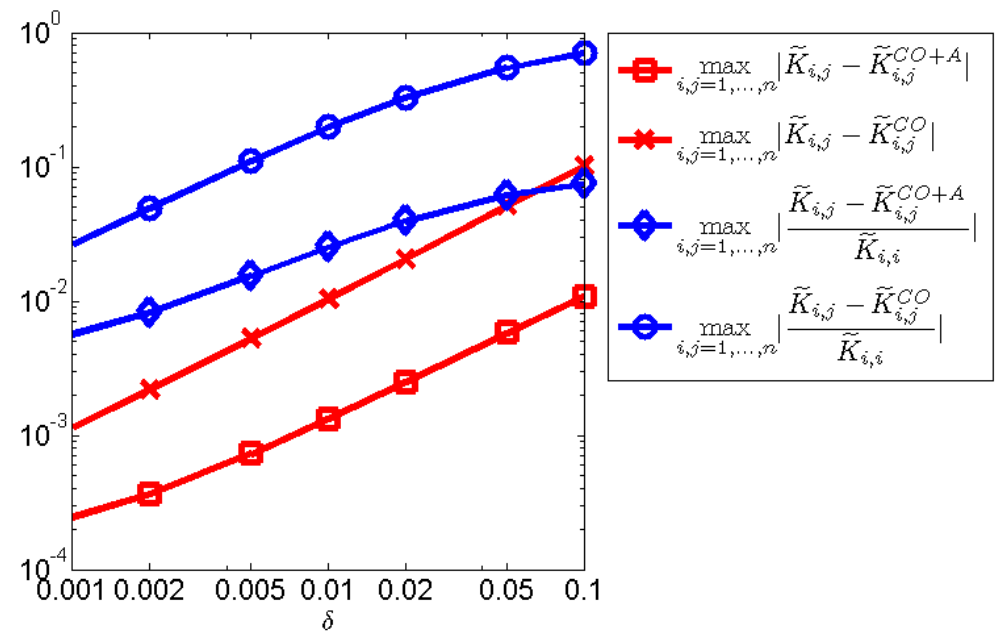

(b) Errors for different contrasts.

FIG. 5.3. Performance of Algorithm 1 for a foam geometry.

\begin{tabular}{|lll|lll|lll|}
\hline \multicolumn{3}{|c|}{$\tilde{K}$} & \multicolumn{3}{c|}{$\tilde{K}^{C O}$} & \multicolumn{3}{c|}{$\tilde{K}^{C O+A}$} \\
\hline 5.27e-02 & $7.90 \mathrm{e}-05$ & $-1.95 \mathrm{e}-04$ & $4.22 \mathrm{e}-02$ & $9.01 \mathrm{e}-05$ & $-2.18 \mathrm{e}-04$ & $5.14 \mathrm{e}-02$ & $9.01 \mathrm{e}-05$ & $-2.18 \mathrm{e}-04$ \\
$7.90 \mathrm{e}-05$ & $5.27 \mathrm{e}-02$ & $1.67 \mathrm{e}-04$ & $8.23 \mathrm{e}-05$ & $4.22 \mathrm{e}-02$ & $1.96 \mathrm{e}-04$ & $8.23 \mathrm{e}-05$ & $5.14 \mathrm{e}-02$ & $1.96 \mathrm{e}-04$ \\
$-1.95 \mathrm{e}-04$ & $1.67 \mathrm{e}-04$ & $5.40 \mathrm{e}-02$ & $-2.14 \mathrm{e}-04$ & $1.80 \mathrm{e}-04$ & $4.35 \mathrm{e}-02$ & $-2.14 \mathrm{e}-04$ & $1.80 \mathrm{e}-04$ & $5.27 \mathrm{e}-02$ \\
\hline
\end{tabular}

TABLE 5.3

Effective thermal conductivity tensors for the foam geometry of Figure 5.3(a) for $\delta=0.01$.

\section{REFERENCES}

[1] B. Aksoylu, I.G. GRAhAm, H. Klie, And R. Scheichl, Towards a rigorously justified algebraic preconditioner for high-contrast diffusion problems. to appear in Computing and Visualization in Science (Special Issue Dedicated to Prof. W. Hackbusch on the Occasion of his 60th Birthday), 2008.

[2] T. ARBOGAST, Analysis of the simulation of single phase flow through a naturally fractured reservoir, SIAM J. Numer. Anal., 26 (1989), pp. 12-29.

[3] T. Arbogast, J. Douglas, JR., And U. Hornung, Derivation of the double porosity model of single phase flow via homogenization theory, SIAM J. Math. Anal., 21 (1990), pp. 823-836.

[4] G.I. Barenblatt, V.M. Entov, And V.M. Ryzhik, Movement of Liquids and Gases in Natural Strata, Nedra, 1984.

[5] A. Bensoussan, J.L. Lions, And G. PAPAnicolau, Asymptotic analysis for periodic structures, Studies in Mathematics and its applications, Vol. 5, North-Holland Publ., 1978.

[6] I. I. Bogdanov, V. V. MourzenKo, J.-F. THOVERT, AND P. M. AdLER, Effective permability of fractured porous media in steady state flow, Water Resources Research, 39 (2003), pp. 1023-1039.

[7] A. Bourgeat And A. Piatnitski, Approximations of effective coefficients in stochastic homogenization, Ann. Inst. Henri Poincaré, Probab. Stat., 40 (2004), pp. 153-165.

[8] S. C. BREnNER And L. R. ScotT, The Mathematical Theory of Finite Element Methods, Springer, 2nd ed., 2002.

[9] R. T. BYNUM, Insulation Handbook, McGraw-Hill Publishing Co., 1st ed., 2000.

[10] R. EWING, O. ILIEV, R. LAZAROV, I. RYBAK, AND J. WILlEMS, An efficient approach for upscaling properties of composite materials with high contrast of coefficients, Tech. Report 132, Fraunhofer ITWM, 2008.

[11] V. Girault And P.-A. Raviart, Finite Element Methods for Navier-Stokes Equations, Springer Series in Computational Mathematics, Springer, 1st ed., 1986.

[12] C. He, M.G. EdWARds, AND L.J. DuRLOFsKY, Numerical calculation of equivalent cell permeability tensors for general 
quadrilateral control volumes, Comput. Geosci., 6 (2002), pp. 29-47.

[13] U. Hornung, ed., Homogenization and Porous Media, vol. 6 of Interdisciplinary Applied Mathematics, Springer, 1st ed., 1997.

[14] O. ILIEV, R. LAZAROV, AND J. WILlEMS, A graph-laplacian approach for calculating the effective thermal conductivity of complicated fiber geometries, Tech. Report 142, Fraunhofer ITWM, 2008.

[15] V. V. Jikov, S. M. Kozlov, And O. A. Oleinik, Homogenization of Differential Operators and Integral Functionals, Springer, 1st ed., 1994.

[16] S. ToRqUATO, Random Heterogeneous Materials. Microstructure and Macroscopic Properties, Interdisciplinary Applied Mathematics. 16. New York, NY: Springer, 2002.

[17] W. TURner, Thermal Insulation Handbook, Krieger Publishing Company, 2006

[18] J.E. WARREN AND P.J. Root, The behavior of naturally fractured rerervoirs, Soc. Petr. Rng. J., 3 (1963), pp. $245-255$.

[19] A. WIEGMANN AND A. ZEMITIS, EJ-HEAT: A fast explicit jump harmonic averaging solver for the effective heat conductivity of composite materials, Tech. Report 94, Institut für Techno- und Wirtschaftsmathematik, 2006.

[20] X. H. Wu, Y. EfendieV, And T. Y. Hou, Analysis of upscaling absolute permeability, Discrete Contin. Dyn. Syst., Ser. B, 2 (2002), pp. 185-204. 